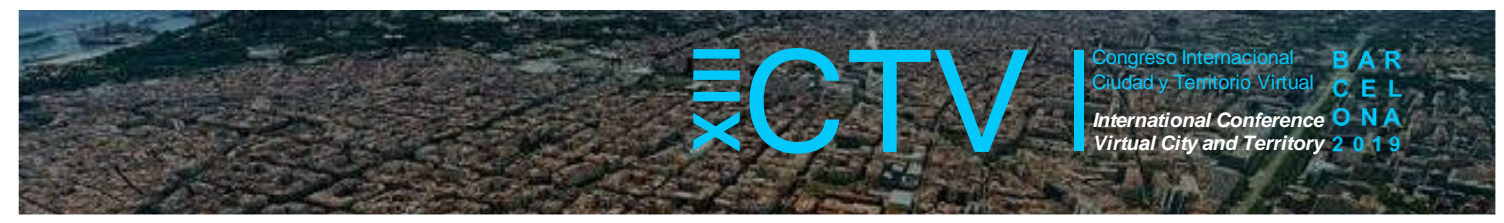

\title{
LOS LÍMITES DE LA CIUDAD: INFRAESTRUCTURA HISTÓRICA RURAL VS. CRECIMIENTO URBANO. EL CASO PRÁCTICO DE L’ORTOLL, EN VILANOVA I LA GELTRÚ
}

\author{
Martínez Görbig, Gerard ${ }^{1}$
}

Remisión inicial: 2019-06-12; Remisión definitiva: 2019-10-28; Publicación: 2019-12-21

Citación: Martínez Görbig, G. (2019). Los límites de la ciudad: infraestructura histórica rural vs. Crecimiento urbano. El caso práctico de L'Ortoll, en Vilanova i la Geltrú. En XIII CTV 2019 Proceedings: XIII International Conference on Virtual City and Territory: "Challenges and paradigms of the contemporary city”: UPC, Barcelona, October 2-4, 2019. Barcelona: CPSV, 2019, p. 8534. E-ISSN 2604-6512. DOI http://dx.doi.org/10.5821/ctv.8534

\section{Resumen}

El crecimiento poco controlado - o nada - de las ciudades de mediano tamaño en las periferias de las metrópolis, o simplemente en medios rurales o agrícolas ha supuesto en numerosas ocasiones la aparición de espacios (¿basura?) poco ortodoxos que confrontan con el tejido impuesto de éstos asentamientos urbanos. Los límites de las ciudades fuera del área metropolitana quedan en una posición conflictiva al enfrentarse con el territorio previamente existente, pues las ciudades mencionadas no tienen fuerza suficiente para enfrontar semejante terreno, y a su vez, porque el sistema de infraestructura de conexión entre ellas, fragmenta y divide el terreno accidentalmente, haciéndole perder a éste su identidad.

La no conexión entre el mundo rural y desurbanizado con las ciudades se convierte en un problema para el crecimiento sostenible del tejido, cuya solución actual debe incluir diferentes conceptos esenciales para el desarrollo territorial contemporáneo, tales como sostenibilidad, tecnología, economía o sociedad. Por tanto, se propone, más que imponer urbanidad en dichas zonas, el incluirlas dentro de los núcleos urbanos, permitiendo así la inclusión de nuevos lienzos que sirvan al desarrollo urbano con espacios verdes, equipamientos, zonas industriales u otros.

Se ha tomado como caso práctico el parque de L'Ortoll, al oeste de Vilanova i la Geltrú (Barcelona), dónde la relación con la ciudad ha quedado negativamente influenciada por el proceso de urbanización, deviniendo una parcela abandonada de grandes dimensiones. Para poder tratar los límites de la zona según lo descrito anteriormente, se ha procedido con un análisis con tres enfoques distintos, utilizando fuentes de los archivos históricos, ortofotografías, tecnología GIS y trabajo de campo, incluyendo la interacción con asociaciones de conservación:

Morfológico y geográfico: evolución de la topografía y del terreno.

Funcional y social: diferentes usos de la zona a lo largo del tiempo.

Histórico, para comprender y asociar los dos anteriores.

Después de realizar dicho análisis, se consigue llegar a diferentes conclusiones, que permiten desarrollar diferentes hipótesis sobre el porqué de la colisión desfavorable:

- El terreno de L'Ortoll pertenece a un sistema mucho mayor que el territorio en sí mismo: la infraestructura perteneciente al sector primario en todo el territorio autónomo catalán.

- El crecimiento rápido e indiscriminado de la ciudad no gestiona sus límites y no puede afrontar la inclusión de otros sistemas ya creados.

- El desarrollo del sistema infraestructural interurbano con Barcelona y Tarragona (principalmente) crea vías rápidas de una velocidad diferente e incompatible con lo rural, que fragmentan el territorio original.

- El sector primario queda disuadido por un sistema económico terciario y secundario de mayor fuerza, dejando obsoleta la zona.

- La necesidad de conservación del interés histórico y natural de la zona colisiona con los planes de urbanización y la especulación de la zona.

- Observamos también dos frentes distintos de actuación versus la zona en cuestión:

- El sector inmobiliario, que intenta especular con el territorio.

- La acción social, que intenta recuperar el territorio, con una tasa de éxito mayor.

Al interpretar lo obtenido, se concluye que para la integración de nuevas zonas con capacidad de dotar la ciudad de espacios verdes, públicos y con posibilidades, de un modo correspondiente a la evolución actual de las ciudades, se necesita trabajar los límites entre tejido urbano como elementos independientes, y posteriormente marcar pautas de

\footnotetext{
${ }^{1}$ Arquitecto por la ETSAB, UPC, * Correo de contacto: gerardm 12@hotmail.com
} 


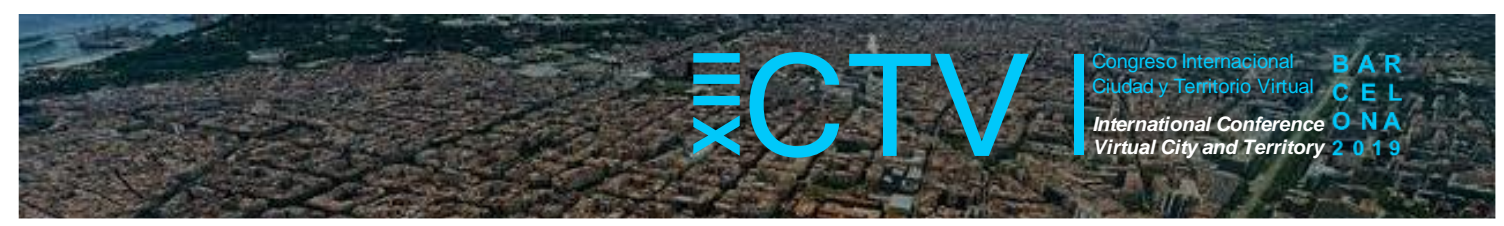

desarrollo para la zona, siempre creando un vínculo con las diferentes capas que tiene la ciudad (física, económica, social, digital, etc.) y el nuevo territorio.

Además, a partir del análisis realizado se intuyen diferentes pistas en las que se deben basar los proyectos en este tipo de territorios para tener éxito:

- La interacción social con el terreno. El propio usuario como generador de ciudad.

- El respeto de lo existente; natural, histórico y urbano.

- El déficit funcional de una zona en concreto.

- La capacidad de crear un sistema con entidad propia, pero permeable a ambos lados.

Finalmente, se intentan aplicar las diferentes conclusiones sobre el terreno con un proyecto de urbanización del límite en cuestión, cuya forma en este ámbito en concreto va a ser un pequeño campus de arqueología, que va a complementar e integrar lo que actualmente ya está ocurriendo en la zona y se extenderá en el territorio en cuestión.

Palabras Clave: límites; urbanidad; rural; integración

\section{Introducción y contexto}

Los límites de las ciudades y zonas urbanas siempre han sido un elemento conflictivo en el desarrollo urbanístico de las mismas. Naturalmente, en grandes ciudades y puntos de interés del territorio siempre ha sido un aspecto controlado, o bien, como mínimo, estudiado.

No obstante, si observamos con detenimiento otro tipo de asentamientos, tales como las ciudades de las periferias de las metrópolis o las que han crecido en un medio rural, nos encontramos con unos contornos conflictivos, generalmente originados por el crecimiento poco controlado de esos mismos asentamientos y su colisión con el medio rural y el entorno que los rodea. Estos espacios, que podríamos llegar a calificar en algunas ocasiones como residuales, son muchas veces fuente de conflicto entre el tejido urbano de la ciudad y el entorno inmediato del mismo, pues no se ha considerado ningún plan para fusionarlos como parte del mismo elemento.

De hecho, el crecimiento esporádico de estas ciudades coincide con el boom económico generado por algún elemento en concreto, ya sea la revolución industrial, la llegada del ferrocarril o el desarrollo de un puerto en ciudades costeras. Naturalmente, este desarrollo económico exprés conlleva también un aumento demográfico y una expansión territorial de la zona, que fluctuará en acorde a lo que lo ha causado. Es por ello que una vez alcanzado el pico de la economía para ese fenómeno concreto, el crecimiento urbanístico que lo acompañaba se frena en seco y se queda sin recursos para seguir desarrollándose adecuadamente, pues no tenemos que olvidar que hablamos de asentamientos no preparados para dicho crecimiento, y que se han dejado llevar por una falsa sensación de euforia de duración determinada.

La falta de potencia (o fuerza) de estos asentamientos resulta en una incapacidad para confrontar un terreno preexistente de mucha mayor fuerza. Además, el sistema de conexión interurbano generado también afecta al terreno preexistente, fragmentándolo y dividiéndolo, y haciéndolo perder su identidad original.

El terreno resultante, que no forma parte de ni del mundo urbano ni del rural, está desconectado. Esta desconexión de tan distintos y cercanos ámbitos se convierte en un problema clave a resolver para las ciudades contemporáneas, pues las cuestiones a tener en cuenta en el desarrollo urbano han evolucionado bastante en los últimos tiempos. El principal hándicap con el que nos encontramos para un crecimiento sostenible del tejido urbano no es 


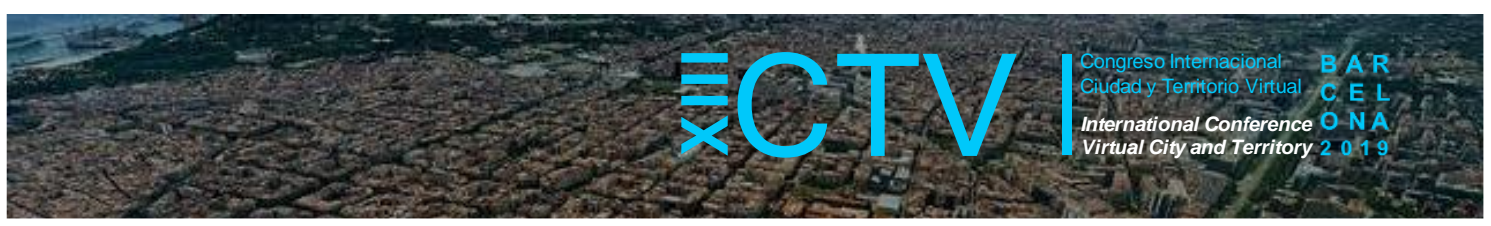

otro que la capacidad en sí de englobar todos los conceptos que forman parte de la ciudad en sí, y que antes se pasaban por alto, como son la tecnología, la economía, la sostenibilidad ambiental o los aspectos sociales y antropológicos.

Por todo ello, resulta interesante proponer la integración de esas zonas en la ciudad desde diferentes puntos de vista, y de una forma fluida que se genere desde la propia ciudad, pero sin imponer planes urbanísticos exentos sobre la zona, pues ello podría conllevar a una desconexión todavía mayor. Es decir, de alguna forma determinada para cada lugar, poder llegar a generar una urbanidad basada en espacios verdes, equipamientos, zonas industriales u otros, a base de entrelazar los diferentes fenómenos que suceden en la ciudad y su entorno.

\section{Análisis, metodología y resultados}

\subsection{L'Ortoll}

Para poder generar una hipótesis consistente sobre esta problemática de los límites, se ha tomado como caso práctico a analizar la zona denominada como L'Ortoll, perteneciente al municipio de Vilanova i la Geltrú, una ciudad costera rodeada por un sistema montañoso a unos $45 \mathrm{~km}$ al suroeste de Barcelona.

Figura 1. Vilanova i la Geltrú respecto Barcelona

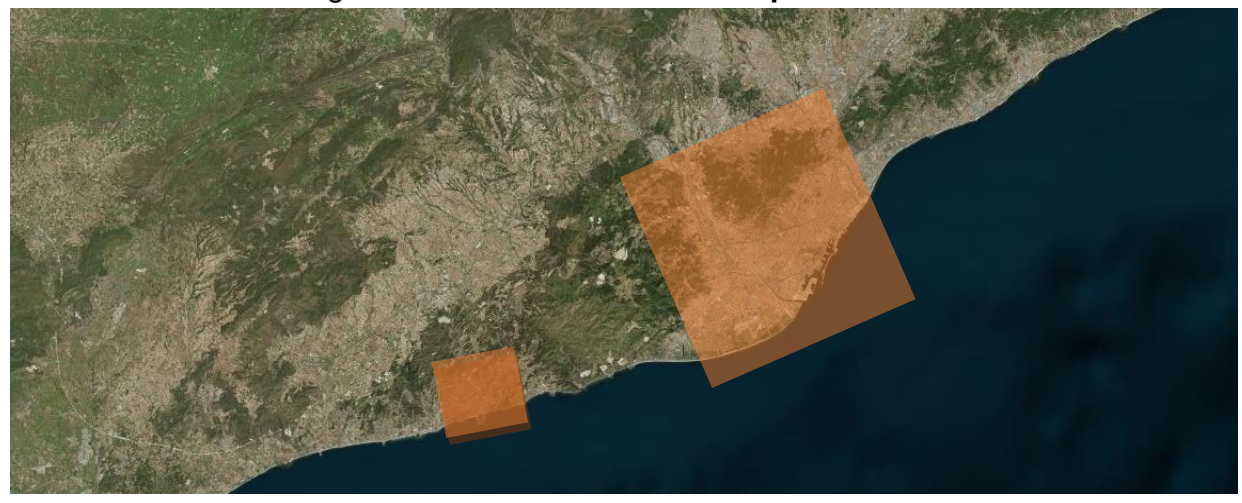

Fuente: Elaboración del autor.

Vilanova i la Geltrú pertenece, a pesar de la distancia que la separa de su capital, al último cinturón del Área Metropolitana de Barcelona, a la zona del Garraf. No obstante, a pesar de estar incluida dentro de esta área, su compleja topografía dificulta el acceso a la misma ciudad y la completa integración urbana tal y como lo hacen ciudades como Castelldefels o Gavà, completamente conectadas mediante el tejido urbano. Es precisamente en estos casos donde la infraestructura interurbana toma un papel de relevancia marcando no tan sólo la rapidez de conexión entre zonas, sino también influyendo en los alrededores inmediatos de la ciudad en cuestión, como veremos posteriormente.

La zona que nos ocupa pertenece precisamente a una gran zona verde preexistente, que debido a diversas transformaciones que ha sufrido ha variado su morfología, función y relación con la ciudad, hasta quedar irreconocible en su punto más próximo a la ciudad, conocida por los lugareños como L'Ortoll. 


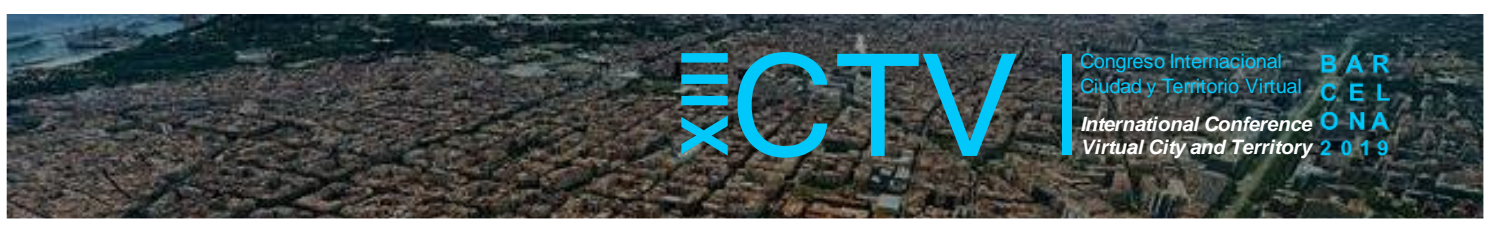

La parcela en cuestión es una extensa masa verde de aproximadamente 97 hectáreas, cuya relación con la ciudad ha quedado prácticamente nula debido al proceso de urbanización del núcleo urbano. Esta desvinculación ha resultado en un abandono del terreno, originando diferentes problemas no sólo de carácter urbanístico, sino también de carácter social y económico. Su gran extensión sin control aparente y sin conexión con la ciudad (y por lo tanto sin vigilancia), hacen de L'Ortoll un lugar propenso para el desarrollo de actividades delictivas, además de la parcela idónea para la instalación de chabolas y otros tipos de urbanizaciones precarias y sin legalizar. Además, la suma de ambas crea un clima de inseguridad en la zona, desvinculándola también de la ciudad en términos sociales. Estos fenómenos no tan solo generaron la rotura con el núcleo urbano, sino que también implicaron el cese de las actividades industriales, pequeños comercios o equipamientos que estaban instalados en la zona en un intento del ayuntamiento local de rescatar la zona (sin éxito).

Figura 2. Vilanova i la Geltrú

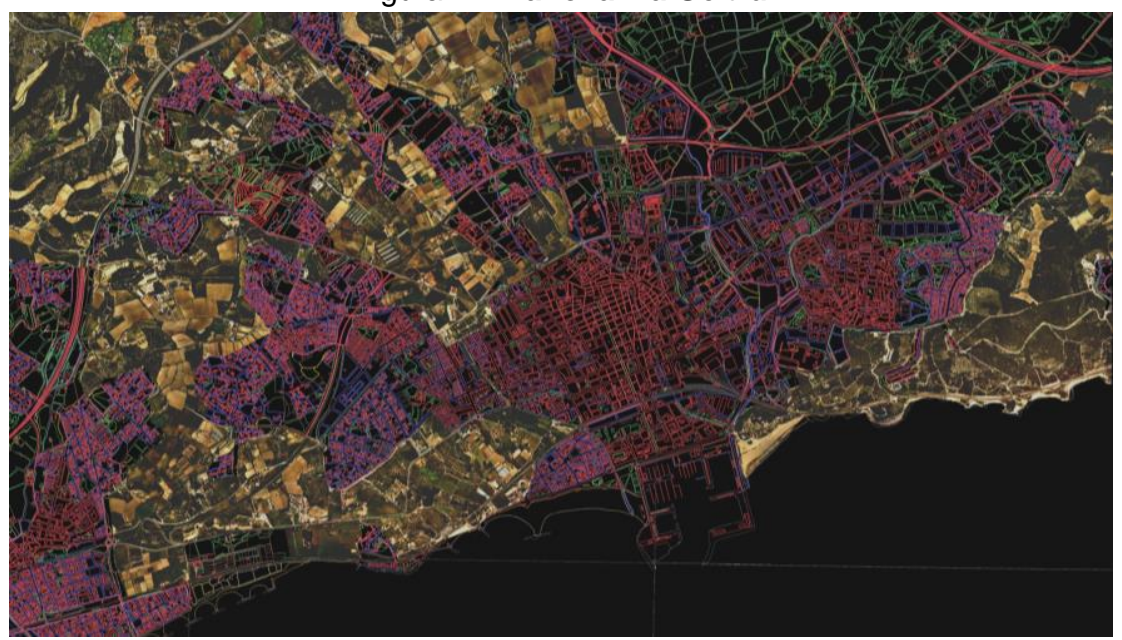

Fuente: Elaboración del autor.

Para poder tratar los límites entre la zona en cuestión y el núcleo urbano, poder incluir todo tipo de cuestiones actuales, y conocer el porqué de la degeneración de la zona, se han realizado diversos análisis del territorio utilizando desde métodos comprendidos desde tecnología GIS hasta el mismo trabajo de campo. Estos análisis permiten conocer con mayor exactitud las peculiaridades del terreno y poder abordar con mayor precisión los puntos de principal conflicto.

Como hemos comentado anteriormente, son varios los factores que influyen en un territorio limítrofe de semejantes características, por lo que se han realizado aproximaciones en el emplazamiento desde distintos puntos de vista que se pueden agrupar en 3 ámbitos: morfológico y geográfico; funcional y social; e histórico.

\subsection{Análisis}

Mediante estos tres enfoques se puede conocer el proceso que ha "condenado" esta zona de ciudad en distintos campos, por lo que no sólo se puede obtener una solución, sino varias que deberán combinarse para poder llegar a un proyecto transversal a todas ellas, y a su vez coherente con los resultados del análisis obtenido. 

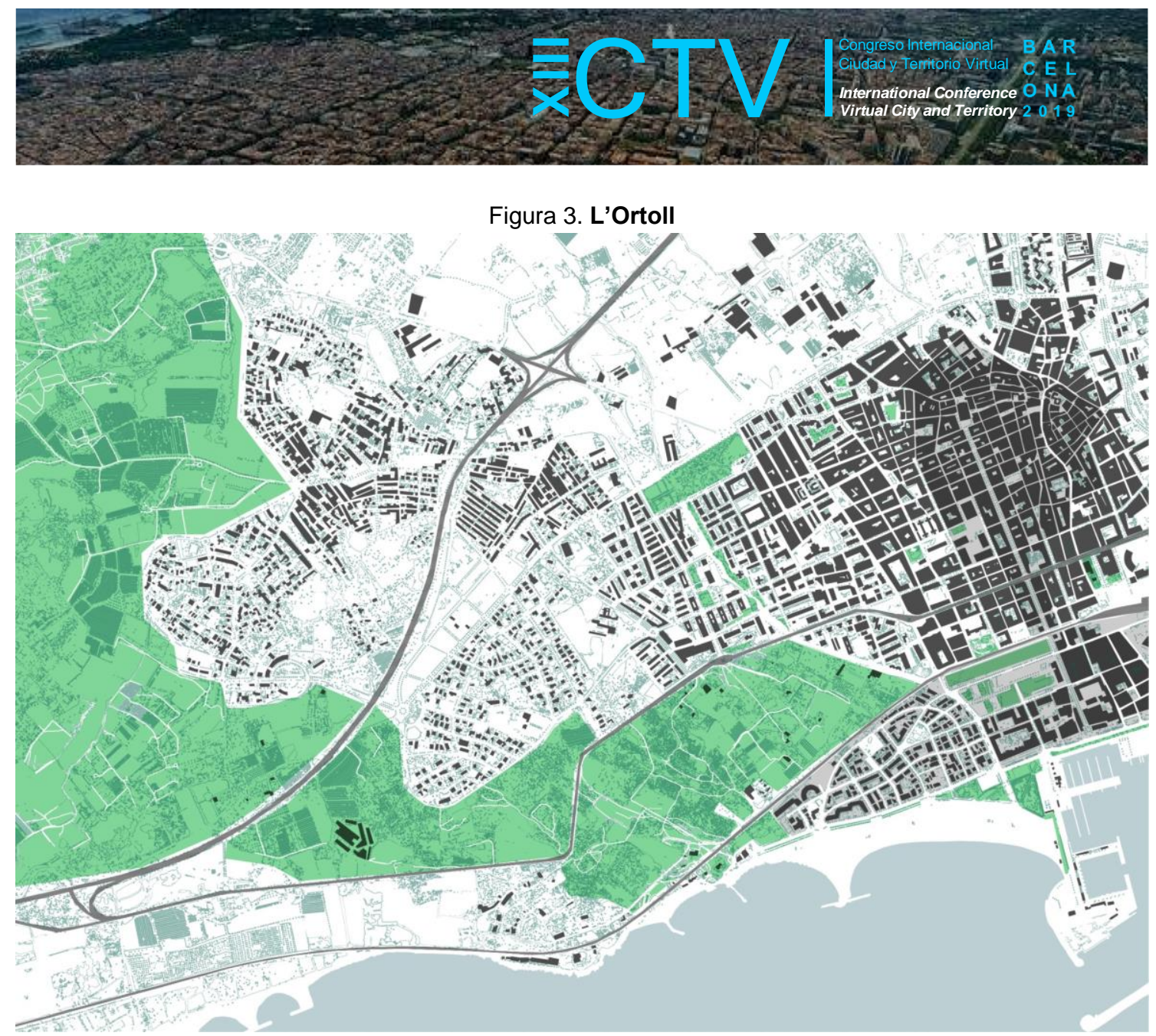

Fuente: Elaboración del autor.

\subsubsection{Morfología y geografía del territorio}

Figura 4. Zona acceso paseo

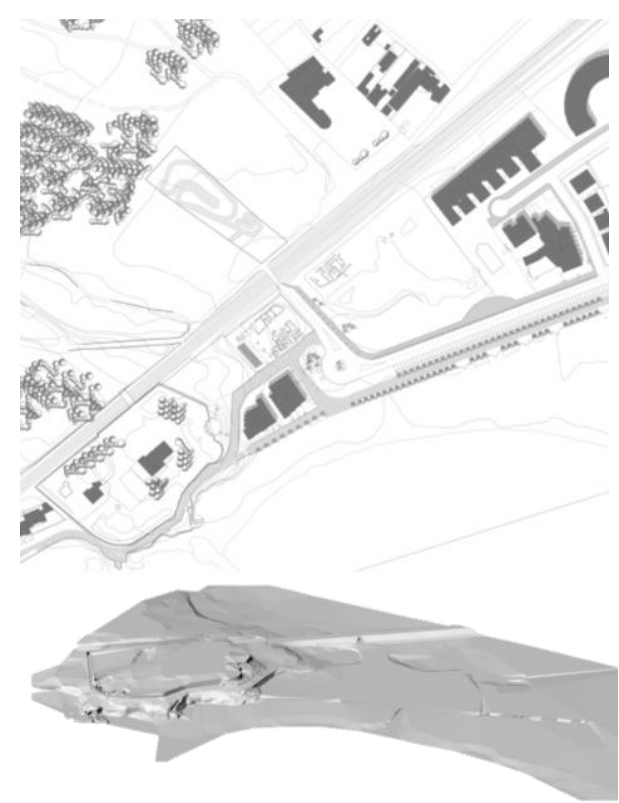

Fuente: elaboración del autor
Nos encontramos con un terreno de topografía compleja. Se trata de un punto donde el sistema montañoso del Massís del Garraf llega a la costa, interrumpiendo así la continuidad de la playa, y generando una zona con cambios de nivel importantes de hasta 40 metros en ciertos puntos, que contrastan con la planeidad del resto del municipio. Además, la aparición repentina de pequeños picos y barrancos costeros genera puntos de difícil acceso y que acaban convirtiéndose en caminos sin salida. Uno de estos puntos es, precisamente, el mayor punto de interconexión entre la zona de L'Ortoll y la ciudad: el Paseo Marítimo de la ciudad. Hacemos ahora un breve paréntesis para acercarnos al Paseo. Se trata de la avenida de mayor afluencia turística de la ciudad después de la Rambla Principal, formando entre las dos un cruce de ejes que organiza la vida interna de la ciudad. No obstante, a medida que nos desplazamos hacia el oeste, la zona playera se ve menguada de servicios y va cobrando forma de ciudad jardín, con 


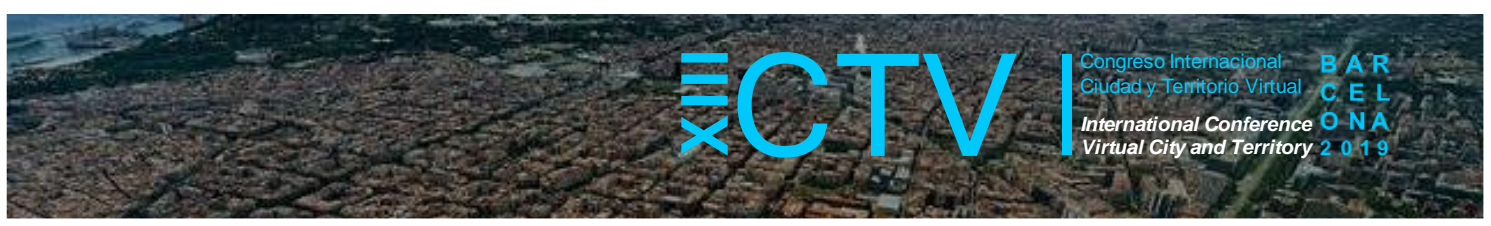

una bajísima densidad y falta de servicios. Si miramos la zona en planta, vemos que la vía del ferrocarril toma una dirección no paralela a la costa, y va cerrando la zona también en esa dirección, dando como resultado un final de paseo cerrado en sí mismo y sin servicios. Es precisamente en ese punto donde se encuentra la entrada a L'Ortoll desde la ciudad, realizada con un paso bajo nivel del ferrocarril.

Si volvemos de nuevo a la zona de L'Ortoll en sí mismo, vemos que la complejidad del terreno y la comparación de los diferentes accidentes que encontramos también nos hablan de las modificaciones contraproducentes que ha sufrido en territorio.

Al tratarse de una zona con laderas, se hallan diversas canalizaciones naturales de agua, principalmente torrentes. Éstos, sobre todo los de mayor calibre, también actúan como una barrera natural entre núcleo urbano y zona rural, generando unos trazos claros que normalmente acompañan algún cambio de nivel. Como apreciamos en las siguientes figuras, todos estos torrentes adoptan una dirección paralela al pendiente natural, pero en numerosos puntos se ven interrumpidos por el sistema de canales agrícolas de la zona, cuya dirección es completamente perpendicular a los mismos, creando pues una inestabilidad en el flujo del agua de la zona.

\section{Figura 5. Torrentes}

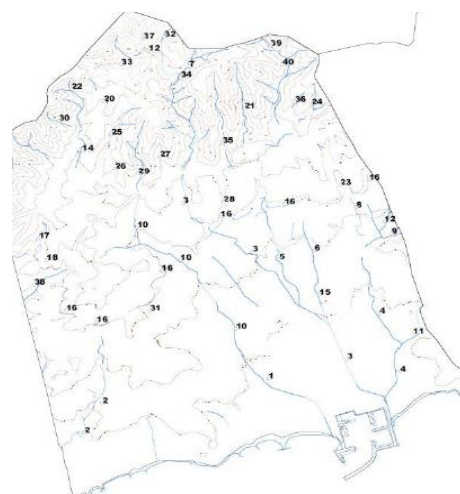

Figura 6. Canalizaciones

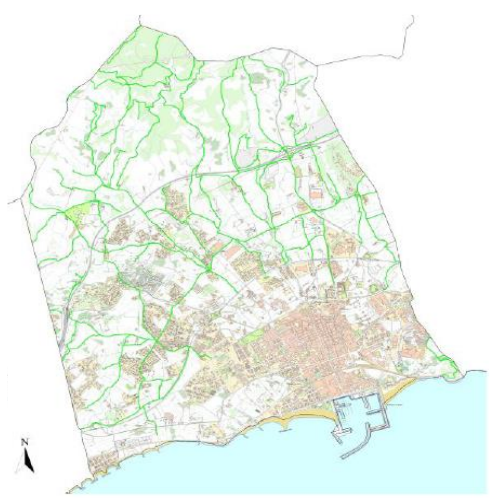

Fuente: elaboración del autor.

También si nos fijamos en la problemática de las preexistencias naturales, debemos tener en cuenta que como en toda zona rural y natural encontramos masas de vegetación extensas que en muchos casos conviene proteger. El hecho de formar parte de un sistema perteneciente al clima mediterráneo implica que muchas de las especies que encontramos en la zona sean protegidas, por lo que es una dificultad añadida a la hora de tratar con el territorio.

Figura 7. Distribución de vegetación

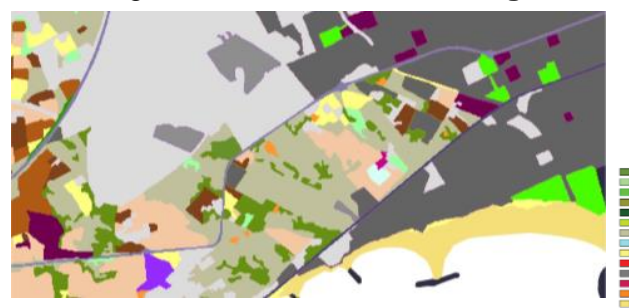

Fuente: ICGC.
Figura 8. Uso del suelo

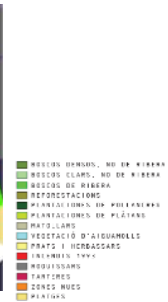

Fuente: ICGC.

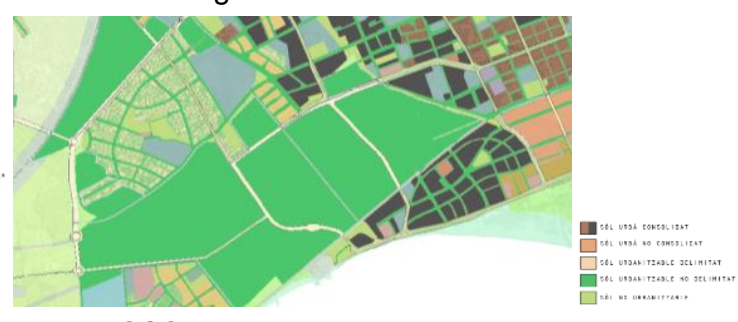




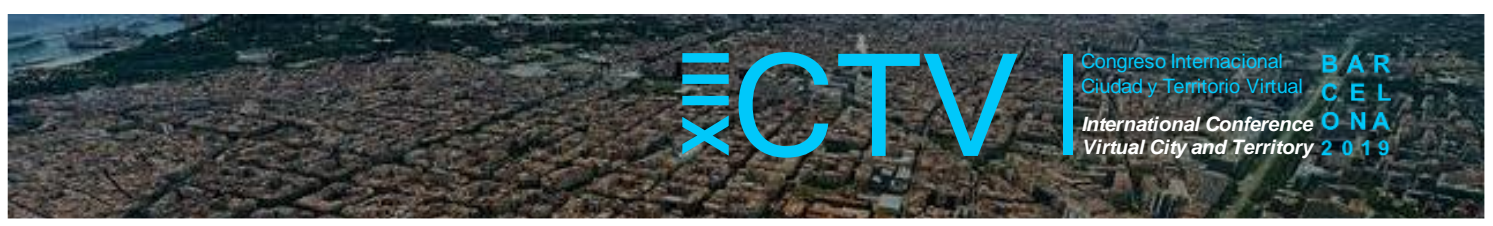

No obstante, el verdadero problema en la morfología del terreno nos lo encontramos cuándo sumamos los accidentes generados a causa de la urbanización. Es decir, aquellos límites físicos que entrecortan el territorio propio del desarrollo urbano, y que principalmente y como hemos dicho anteriormente, forman parte del sistema de conexión interurbana del territorio. Encontramos tres tipos de barreras: la vía del ferrocarril, las carreteras y la autovía.

Figura 9. Caminos

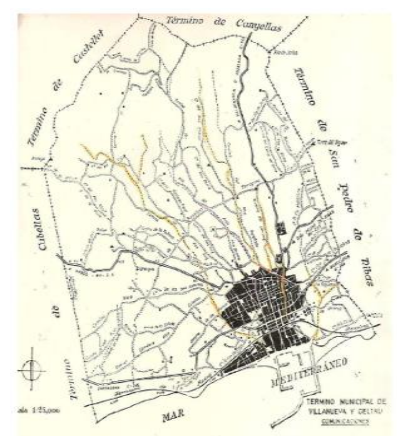

Figura 10. Vías rápidas

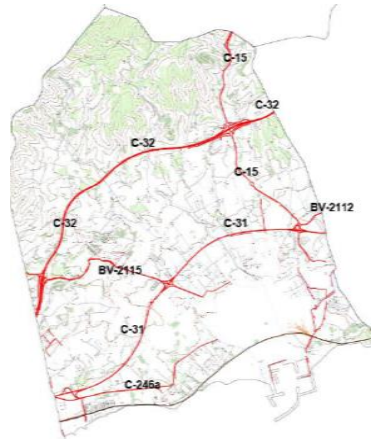

Fuente: elaboración del autor.

Estos tres elementos fragmentan el terreno creando una frontera muy clara que limita y encierra en sí mismo el terreno, pues son vías de velocidades muy mayores de las que puede adoptar la zona rural. Además, en el caso de la vía ferroviaria también se genera una barrera visual que impide cualquier tipo de relación entre ciudad y la zona en cuestión.

Si analizamos estas barreras en sí mismas, vemos que la autovía y la carretera seccionan el territorio por el norte, separándolo de lo que sería su continuación directa hacia el sistema montañoso al que pertenece, por lo que deja de formar parte directamente de su propia entidad. A su vez, la vía ferroviaria corta las posibles conexiones con la ciudad y la zona de la playa, dejando tan solo un pequeño paso bajo la vía en el extremo occidental del paseo marítimo.

Es curioso observar también que antes de la aparición de las vías de velocidad rápida ya contábamos con numerosos caminos interurbanos en la zona. No obstante, en aquel entonces los caminos seguían el flujo natural de la masa verde, siendo coherentes con la topografía. Es tan sólo en la aparición de las vías rápidas generadas durante la segunda mitad del siglo XX dónde encontramos la mayor capacidad de segregación.

Para finalizar, y no menos importante, tenemos la importancia de la geometría generada por los límites. Al no tratarse de planeamientos paralelos o perpendiculares (recordamos que las vías interurbanas y la zona urbana en sí se genera a partir de un crecimiento espontáneo y poco controlado), encontramos cantidad de ángulos agudos que sumados a la sección topográfica del terreno resultan en parcelas angostas y poco agradecidas para una integración de un plan urbano convencional.

\subsubsection{Funcional y social}

Funcional y socialmente nos encontramos ante un terreno con actividad prácticamente nula desde los años 80 hasta 2011, cuando la acción de algunas asociaciones en la zona empezó a 


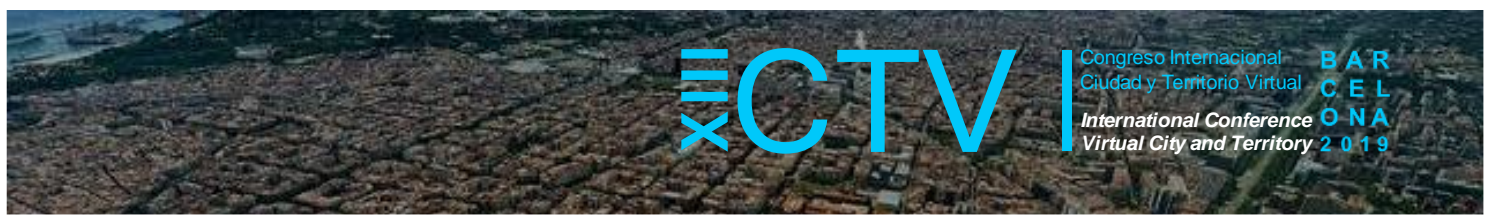

aparecer. En lo que a planes urbanísticos se refiere, en la actualidad tan sólo encontramos un equipamiento de escuela primaria pública, unas naves industriales de pequeño tamaño sin uso, actividades agrícolas y un karting abandonado, todo ello resultante de planes urbanísticos realizados en la zona con intención de reactivar el parque.

En referencia a actividades sociales, encontramos dos tipos de sistemas diferenciados que cohabitan en el territorio. En primer lugar y en menor escalara, encontramos una pequeña urbanización ilegal de chabolas, con una actividad cerrada en sí misma, como si de un sistema aislado de tratara. En contraposición, y actuando como un sistema abierto e integrador, encontramos diferentes actividades sociales de recuperación de la zona, de estructura totalmente horizontal y basadas en la colaboración entre vecinos y voluntariados, que actúan en la totalidad de la zona restaurando las preexistencias que allí se encuentran.

Si analizamos el sistema de caminos internos con perspectiva, y no tan sólo en la parte que nos ocupa, se observa que nuestra zona se conecta directamente con otros muchos puntos alejados de la topografía catalana, como los Pirineos o el delta del Ebro, con rutas naturales y rurales entrelazadas a lo largo del territorio. A pesar de encontrar hoy en día poca actividad en la zona, anteriormente al crecimiento de la ciudad y su colisión, L'Ortoll era una zona muy importante dentro del sector primario, pues forma parte del sistema de caminos y rutas de la trashumancia en Cataluña, siendo el punto más costero de éste, y por lo tanto un punto de crucial importancia en el sistema, ya que era la destinación principal en épocas de baja temperatura. No es de extrañar pues, que L'Ortoll fuere una de las zonas más concurridas debido a su proximidad a Barcelona y su entorno costero, natural y aislado.

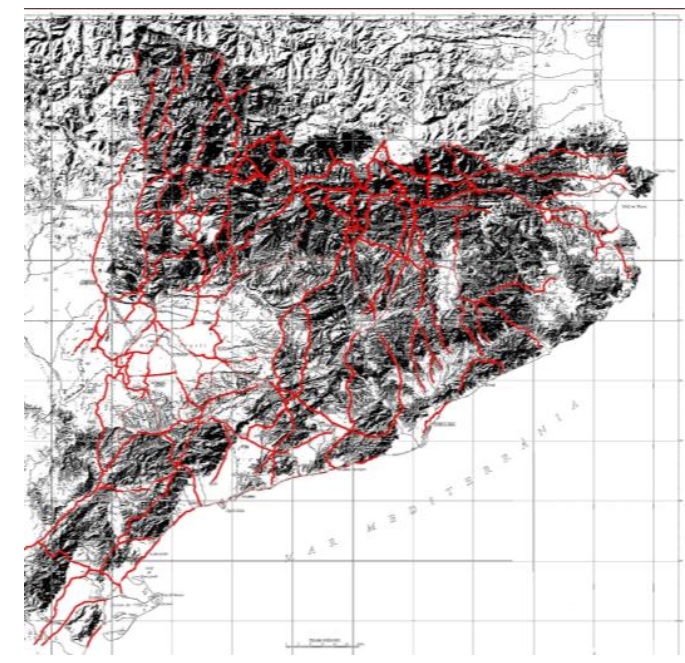

Figura 11. Sistema de caminos de trashumancia en Cataluña Fuente: ICGC
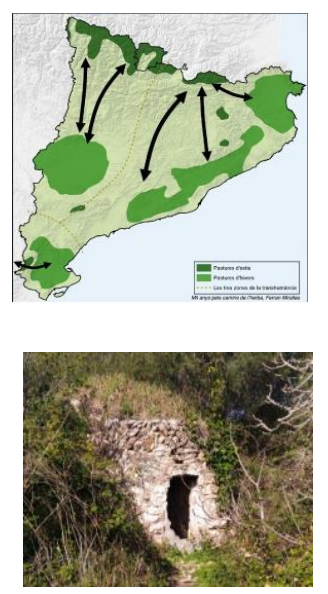

Resulta interesante pues, el contraste entre sector primario y sector terciario que se genera en la zona, y como claramente la colisión de dos sectores tan potentes ha resultado en una segregación del terreno rural y la ciudad, pues la potencia del sector primario preexistente ha impedido a la ciudad integrarlo dentro de su tejido, y éste ha acabado muriendo por sí mismo, dejando atrás numerosas edificaciones

rurales, ruinas o formaciones de caminos de piedra seca de interés que tienen un impacto directo en el carácter de la zona, y cuya importancia histórica resulta innegable. 


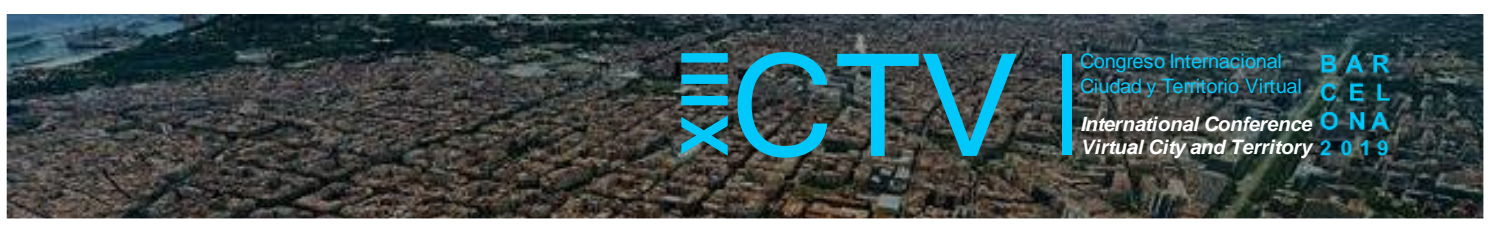

Además, paralelamente a las ruinas del sistema rural, en la zona de la playa, encontramos las ruinas pertenecientes al poblado íbero d'Adarró, de evidente interés histórico, que se encuentra protegido y en excavaciones. Originalmente, la zona estaba destinada a la construcción de complejos de apartamentos en primera línea de mar, pero durante las excavaciones y el movimiento de tierras pertenecientes se hallaron dichas ruinas, provocando otra vez un giro en la funcionalidad y uso de las parcelas.

Por lo tanto, la conexión de la zona verde con la playa a través de la vía del tren acaba siendo también en este campo un punto de conflicto, pues hallamos unas parcelas no edificables y protegidas de propiedad privada, en las que hacer una inversión para seguir con el tejido de la ciudad sería un negocio claramente deficitario.

Si analizamos detenidamente una sección de terreno concreta, donde se conectan ciudad y territorio rural, apreciamos también en términos de funcionalidad el porqué de la dificultad en realizar una conexión coherente: prácticamente el $40 \%$ de la zona está ocupada por parcelas de ocupación privada, de vivienda o simplemente sin edificar; el $32 \%$ de la zona es terreno no urbanizable, pero a su vez sin uso; el $12 \%$ lo ocupan espacios de equipamiento, como la escuela; el $10 \%$ lo ocupan las naves industriales sin uso; el $4 \%$ espacio de interés histórico reconocido, mientras que el $1 \%$ restante es espacio destinado al uso comercial.

Como apreciamos, también en la distribución por usos no resulta buena para realizar una zona de conexión y actividad, ya que, si además nos fijamos en la distribución de los usos en el territorio, vemos que la segregación también en ese aspecto es evidente.

Como puntos relevantes a tener en cuenta, vemos que para un área rural el tráfico rodado a motor toma una importancia desmedida en la conexión, mientras que el ámbito peatonal se queda restringido a dentro de la ciudad. Las zonas de interés y equipamiento son muy reducidas respecto al global, mientras que espacio privado o en blanco predomina en la zona.

Figura 13. (a) Tráfico - (b) Equipamiento - (c) Peatonal

- (d) Plaza - (e) Interés - (f) Agua

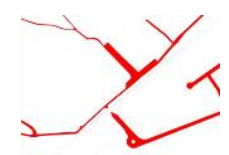

(a)

(b)

Fuente: elaboración propia.

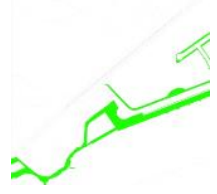

(c) (d)

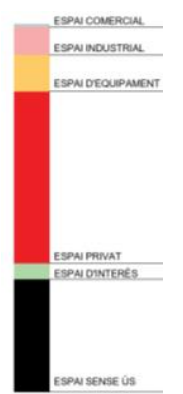




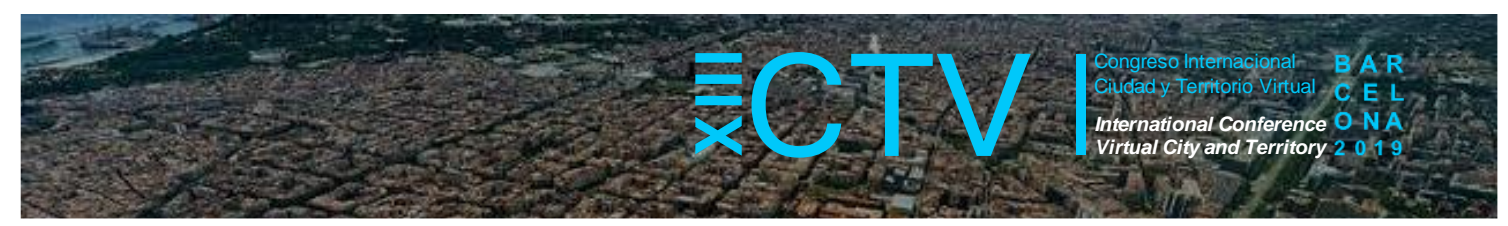

\subsubsection{Histórico}

Para llegar a comprender el porqué de este crecimiento de la ciudad de forma irregular que ha causado los puntos descritos anteriormente, debemos realizar un análisis en retrospectiva de lo sucedido en esta zona desde su formación.

Actualmente la ciudad abarca una zona de aproximadamente $34 \mathrm{~km} 2$, con una población de 66.077 habitantes (2017). No obstante, si nos centramos en la evolución del último siglo, esta ciudad ha multiplicado por seis su población, y prácticamente doblado su área, conllevando también un aumento de la densidad de población.

Al igual que muchos otros municipios próximos a la capital, esta evolución está marcada por la llegada de diversos factores, sobretodo económico, que tuvieron un gran efecto sobre la ciudad. Estos factores no eran otros que la revolución industrial, que buscaba en estas ciudades cercanas terrenos más económicos donde asentarse y poder expandirse. El beneficio por eso, era mutuo, dando a los gobiernos locales una inyección económica importante que se traducía en una inversión en el crecimiento de la ciudad, de forma rápida, para poder alojar a los trabajadores.

En Vilanova i la Geltrú la principal fuente de ingresos no fue otra que la llegada del ferrocarril, la ampliación de su puerto y la llegada a la ciudad en 1902 de la gran empresa multinacional Pirelli S.A. (hoy en día conocida como Prysmian, S.A.), que realizaría una de sus mayores inversiones allí, siendo la primera multinacional en instalarse en dicho territorio.

Esta industrialización súbita de la ciudad y su debida inyección económica y crecimiento, chocó con lo que era todavía en aquel entonces uno de los principales motores económicos de la ciudad, el sector primario. Pese a llevar cerca de los 50 años en un proceso de industrialización basado en la industria textil, la llegada del sector secundario todavía no se había establecido con fuerza en la zona. Para entonces y como ya se ha comentado anteriormente, L'Ortoll tenía una gran importancia en el sector primario y por ello, se conservó.

Con el paso de los siguientes 20 años, aproximadamente hasta 1970-1980, la economía del lugar estuvo en auge, pero a su vez se iba estabilizando de forma natural, de forma que las curvas de crecimiento de la ciudad fueron tomando una pendiente más horizontal, reduciendo así la capacidad de crecimiento de la ciudad, manteniendo L'Ortoll como una zona con su capacidad propia, y quedándose fuera del tejido urbano. En este punto ya teníamos entonces una clara segregación entre medio rural y medio urbano, en términos de morfología y uso del terreno.

El paso definitivo para el abandono de la zona de L'Ortoll sucedió a partir del auge del sector terciario en la ciudad. Las ciudades costeras cercanas a Barcelona encontraron a finales del siglo XX una fuente de inversión fiable en el turismo y en ser ciudades residenciales. Esta inversión en el sector desplazó definitivamente el sector primario fuera de la ciudad, y terminó de segregar las masas verdes con nuevas inversiones en infraestructuras de conexión con la capital, dejando la zona de L'Ortoll como una gran parcela verde sin un uso definido.

En la historia reciente, el Ayuntamiento de la ciudad ha empleado diferentes medidas para poder reactivar la zona, pero sin éxito. Algunos de los ejemplos de planes urbanísticos 


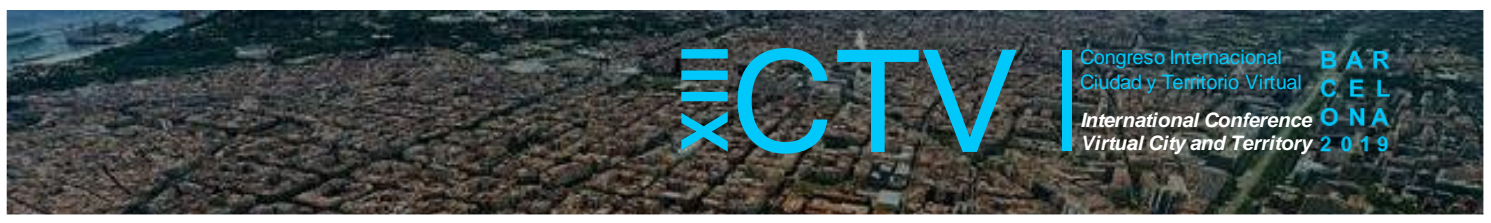

realizados o planeados son la creación de una escuela primaria, todavía activa; proporcionar ayudas a los agricultores y empresas que se encuentran en la zona, sin una repercusión clara sobre la ciudad; o la creación de circuitos de ocio o deportes, como un circuito de BTT o la construcción de un karting, ambos en desuso al cabo de pocos años.

Por otro lado, en 2011 nace la asociación Defensem L'Ortoll, que decide hacerse cargo de la zona en un inicio, con voluntariados. Esta iniciativa cogió fuerza con el paso de los años, pues no tuvo oposición alguna y era perfectamente coherente con el terreno. En 2017, la Plataforma se consolidó en el territorio definitivamente realizando un primer campo de trabajo internacional para estudiantes y trabajadores, con grandes avances como resultado, pero además con un fuerte carácter formativo y práctico en el campo de la restauración y la arqueología. Hoy en día la iniciativa sigue activa con reuniones semanales con voluntariados, y anualmente se sigue celebrando el campo de trabajo.

Figura 14. Evolución histórica de la ciudad
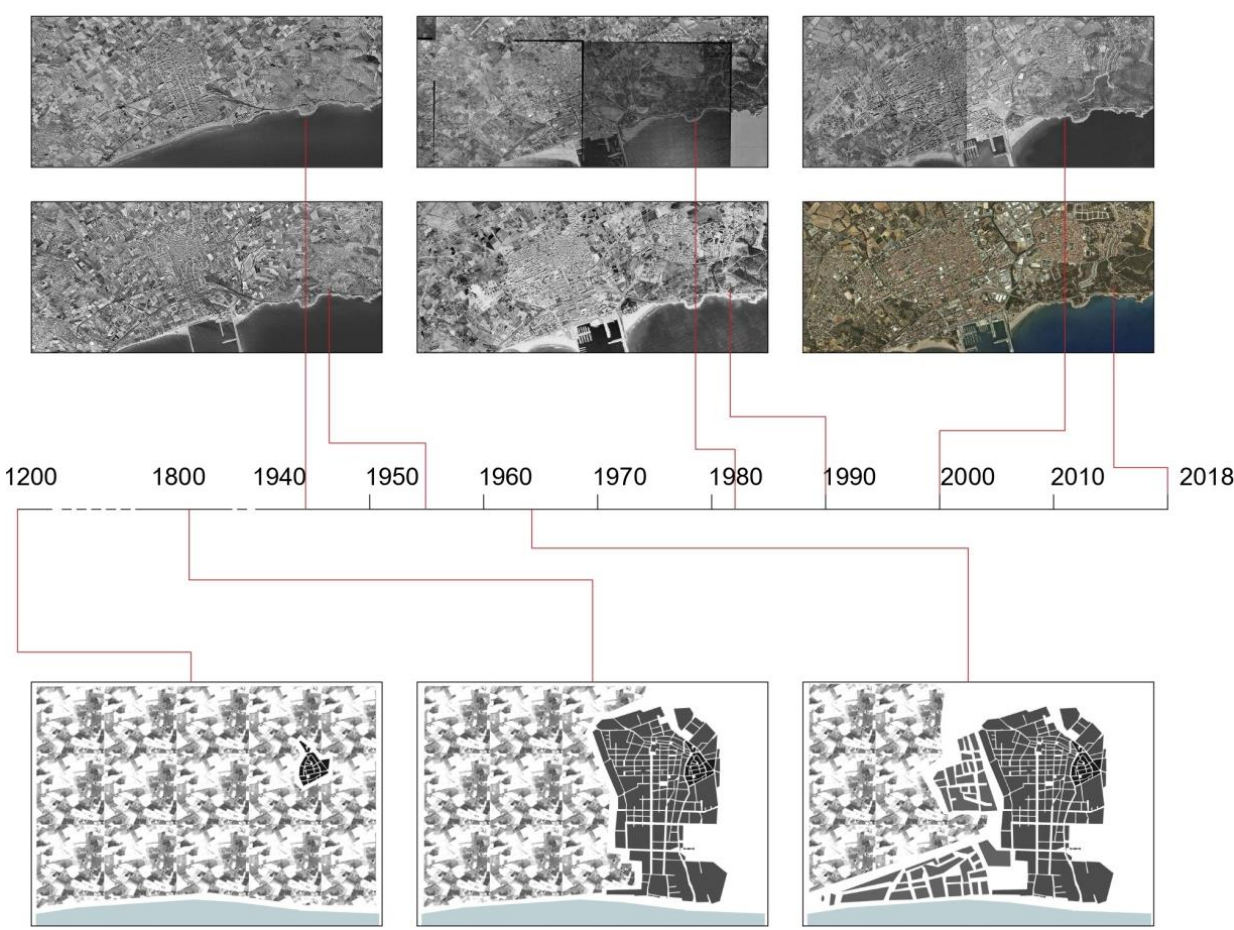

Fuente: elaboración del autor.

\section{Resultados y conclusiones}

\subsection{Resultados}

Con los resultados obtenidos en el análisis, se ha realizado junto a la opinión ciudadana una interpretación sobre los porcentajes de uso y distribución del proyecto que conllevan, junto con estrategias de conservación determinadas para un punto en concreto. 


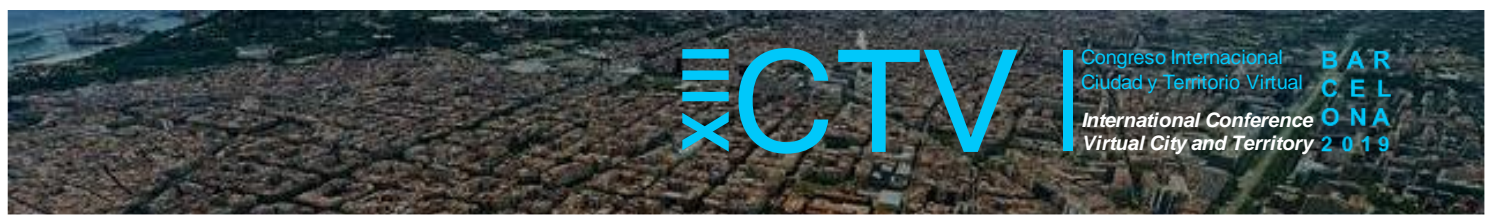

Respecto a los nuevos usos del territorio, es conveniente pensar en una solución correspondiente a lo existente, con su respectiva distribución con el territorio, pero siempre teniendo en cuenta la posibilidad de realizar nuevas edificaciones o zonas para poder complementar su función.

En primer lugar, la redistribución de la barra de usos debe dirigirse claramente a una mayor importancia de las zonas de uso público y equipamientos, que conlleve a su vez una reducción del área sin uso y conflictiva. De la anterior distribución, se pasa a una barra de usos que mantiene el $40 \%$

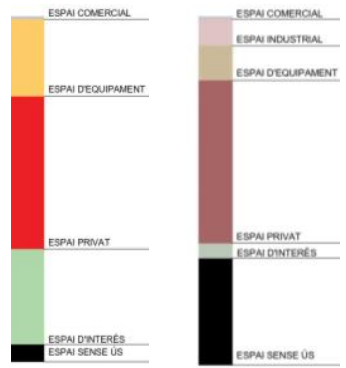

Figura 15. Redistribución de usos de propiedad privada y el $1 \%$ de uso comercial, pues dichas propiedades son inamovibles.

El resto de espacio desfavorable queda considerablemente reducido, dejando al espacio sin uso en tan sólo en un 9\%, y suprimiendo las zonas de carácter industrial. En referencia a los espacios que se podrían considerar favorables para el desarrollo urbano, como son los equipamientos y las zonas de interés (histórico y natural), aumentan su ocupación hasta un $22 \%$ y $28 \%$ respectivamente.

Trasladando dicha barra en un planeamiento formal, obtenemos las siguientes modificaciones en el territorio:

Figura 16. (a) Tráfico - (b) Equipamiento - (c) Peatonal

- (d) Plaza - (e) Interés - (f) Agua

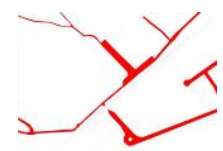

(a)

(b)

Fuente: elaboración propia.

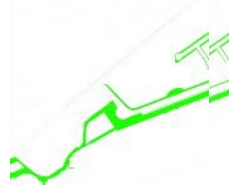

(c) (d)

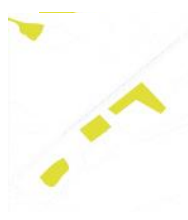

(e)

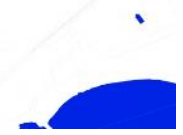

(f)

Apreciamos claramente un aumento en la propia zona de lugares de interés, a la vez que aumenta también el espacio público. Paralelamente, en lo que a movilidad se refiere, obtenemos una reducción del tráfico rodado dentro de L'Ortoll, habilitando tan sólo una vía de conexión con una de las carreteras de mayor importancia a través de la parcela. Para los peatones, bicicletas y otros vehículos de distinta movilidad, se prevé la restauración de los caminos históricos haciéndolos aptos para ellos. De este modo, queda reactivado (por lo menos formalmente) el tráfico interno de L'Ortoll con las velocidades idóneas para convertirlo en una zona verde integrada en la ciudad y con uso para los ciudadanos.

Fijándonos ahora exclusivamente en los resultados del análisis social de la zona, se aprecia claramente que el único agente capaz de dar indicios de reactivación en la zona ha sido la movilización de los vecinos y las plataformas de voluntarios. 


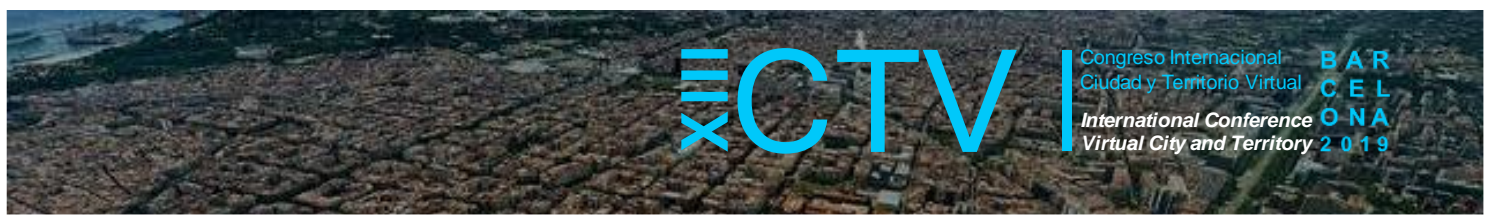

Siguiendo por ese camino, y siendo clara la intención de aumentar las zonas destinadas a equipamiento para aportar una actividad al lugar, se decide adoptar en este punto la colocación de tres equipamientos distintos que ayuden a desarrollar la actividad en la zona y que puedan seguir con el camino marcado.

Para ello, se realizan distintos análisis de actividad anual en diferentes casos de estudio de diferentes tipologías de equipamientos. El resultado gráfico final de los tres escogidos nos muestra que ninguno de los analizados tiene una actividad continuada a través de los doce meses del año, pero no obstante la combinación de los mismos y una posible versatilidad puntual de ellos nos aportan una solución de actividad estable a lo largo del año.

Los tres equipamientos escogidos son: un albergue, una escuela de formación de restauración y un centro cívico. Las tres tipologías de

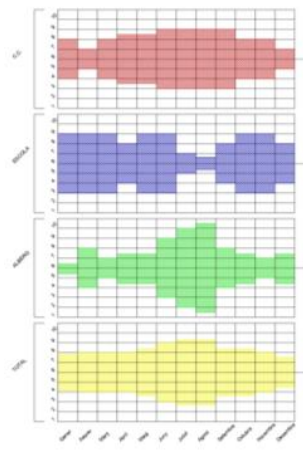

Figura 17. Diagrama usos edificios se complementan para formar una tipología de urbanización de campus con un uso prácticamente enfocado a la restauración, distribuido a lado y lado de la vía del tren en la zona del paseo. Mientras que la escuela de formación actúa como catalizador del campus, el albergue permite crear un flujo constante de movimiento de un lado de la ciudad a otro, y el centro permite la conexión del trabajo realizado con la ciudad en sí.

Como estrategia de emplazamiento del nuevo equipamiento se usa la interpretación de la información obtenida del análisis morfológico de la zona. En primer lugar, detectando la zona de entrada con el paseo se identifica como el principal punto de entrada. Actuando sobre la zona, se detectan las diferentes direcciones que conforman la geometría del territorio para poder implantar un sistema ordenado en todas las direcciones. Seguidamente, teniendo en cuenta los puntos de articulación principales se establecen las localizaciones concretas de los edificios del campus, teniendo en cuenta diferentes estrategias de conexión visual y de movilidad entre ellos.

Figura 18. Estrategias de implementación
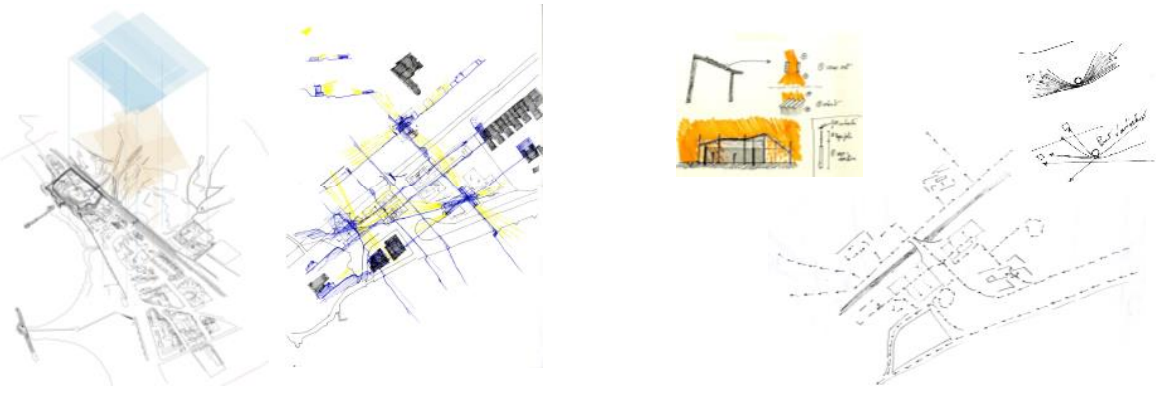

Fuente: elaboración propia.

La formalización final de los edificios corresponde a diferentes estrategias de diseño arquitectónico de carácter sostenible y eficiente, siguiendo la evolución en el mundo del urbanismo y arquitectura con la que convivimos. 


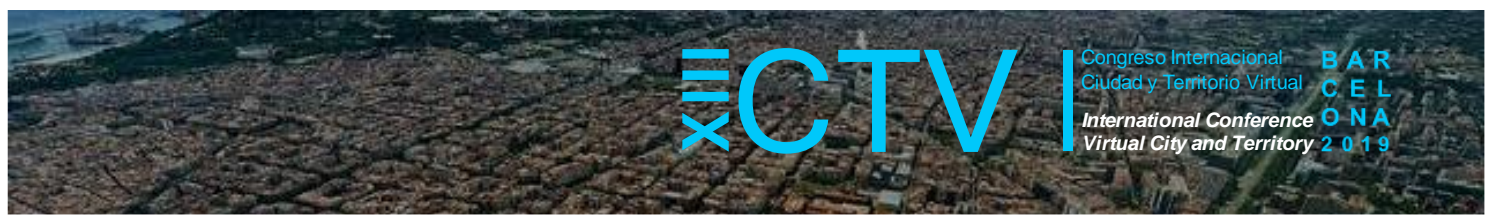

Figura 19. Campus de arqueología

Fuente: elaboración propia.

\subsection{Conclusiones}

El análisis y observación de la zona junto con su resolución teórica, nos muestra que la colaboración ciudadana y la elaboración de una estructura social diferente actuando directamente sobre las preexistencias del territorio tiene una efectividad asegurada. $\mathrm{Si}$ analizamos el contexto real de la zona observamos que, a pesar de los múltiples intentos de urbanización del territorio, no ha sido otra que la acción ciudadana la que ha logrado, sin un plan urbanístico definido, iniciar y proseguir con la restauración de la zona.

Los dos motivos principales explicando este fenómeno no son otros que:

- El territorio preexistente tiene una inercia determinada, y sin una actuación invasiva no se puede colonizar la zona. Para actuar con éxito sobre un territorio se deben seguir dos estrategias opuestas como son la actuación invasiva sobre el territorio, o bien la actuación en puntos determinados para crear nexos de unión con la ciudad.

- En ambos casos no debemos olvidar que la ciudad la forma el ciudadano. A pesar de tratarse de un tópico evidente, la creación de una expansión de la ciudad a partir del flujo social no es, en este caso, banal. Debido a la imposibilidad de imponer un plan urbanístico claro, el fenómeno de la re-activación social de un territorio debe ser primordial es anterior a la implantación de un plan de ordenación, para asegurarse del éxito del mismo.

En estos casos, por lo tanto, es importante reconocer el urbanismo como un fenómeno de carácter social y organizador. En el caso concreto de L'Ortoll ha llegado a rehabilitar una zona de 97 hectáreas que llevaban 40 años en desuso en un periodo de 8 años con iniciativas locales e internacionales. Además, ha logrado integrar con éxito los sistemas aislados que se encontraban en la parcela en un sistema mayor de integración con la ciudad. La siguiente conclusión sobre la formalización de la planificación urbanística en sí, también debe segregarse en dos puntos distintos. 


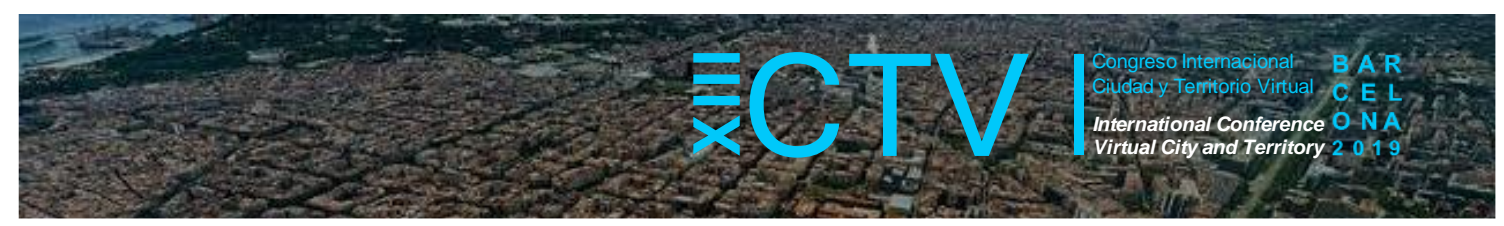

- La implantación de un plan urbanístico determinado debe ser coherente con la fuerza del territorio, pero no imponerse sobre él. Se debe actuar en estos casos desde un punto de vista de la integración de una zona con otra, realizando un proyecto focalizado en puntos concretos localizados en los límites de las ciudades y que actúen como catalizadores de actividad, con posibilidad de expandirse. La función debe predominar, en este caso, sobre el resto de aspectos propios de la arquitectura.

- En caso de haber la necesidad de un plan urbanística, se recomienda la implantación de una tipología de campus. Se considera que la flexibilidad que nos ofrece el sistema urbanístico propuesto es idónea para la creación de espacios de interconexión, pues una de las principales características de dicha tipología es la conexión directa entre sus edificaciones mediante el uso de los mismos, la capacidad de crear un sistema limitado en su función, pero que a su vez se muestra físicamente abierto, y permite la creación dentro del mismo de espacios públicos flexibles y de circulación abierta.

No obstante, en el momento de la elaboración del mismo plan se deben considerar los limites inmediatos de las zonas como parte propia del campus.

Como última conclusión respecto al estudio en cuestión, es importante resaltar que la problemática está presente en numerosas de ciudades de mediano tamaño en países desarrollados y en desarrollo. La rápida expansión de la economía industrial a lo largo de la última mitad de siglo ha conllevado a la creación de dichos espacios conflictivos. En la construcción de infraestructuras interurbanas cerca de los núcleos urbanos se debe siempre seguir unas pautas de análisis y desarrollo que aseguren la integración de las nuevas parcelas creadas, llegando a recomendar la implantación de planes urbanísticos complementarios entre el ámbito supramunicipal y el municipal en sí mismo, fenómeno que no ha sucedido en el desarrollo asociado con el crecimiento económico súbito de algunas ciudades y metrópolis. La confrontación directa con el terreno siempre ha sido un tema a tener en cuenta, pero hoy en día nos encontramos ante un escenario donde la integración de múltiples variables en el terreno debe ser una prioridad, tales como la sostenibilidad, la tecnología, el ámbito social, educativo, comercial, la eficiencia energética, etc. No se debe obviar que la humanidad (y las ciudades) se encuentran en una época de crisis ambiental donde el desarrollo urbanístico sostenible es necesario para la encontrar solución al crecimiento urbano actual, teniendo en cuenta todas las variantes que se han mencionado anteriormente.

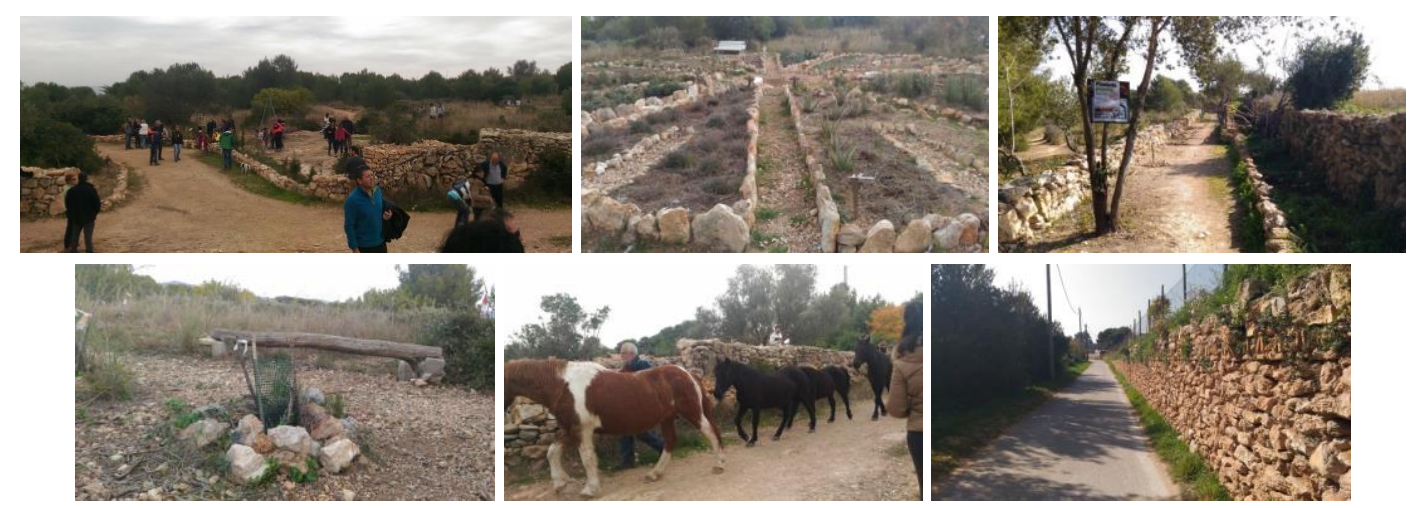




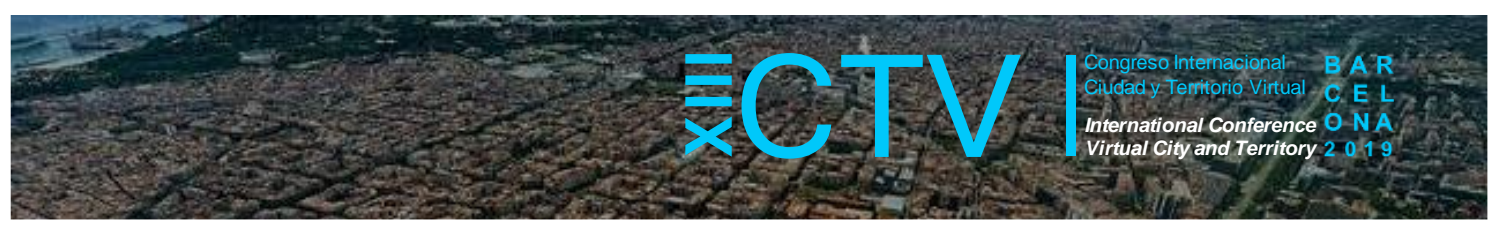

Agradecimientos: Plataforma Defensem L'Ortoll, por su labor. Dep. Proyectos ETSAB, por su tutorización en el inicio de la investigación. Prof. Ángel Martín Ramos, por su incentivación a seguir con el proyecto.

Contribuciones de los autores: Martínez Görbig, Gerard. Elaboración investigación y artículo.

Conflicto de Intereses: Los autores declaran que no hay conflicto de intereses.

\section{Bibliografía}

Copernicus. www.land.copernicus.eu

Geipel, Finn (2009). Grand Paris/Metropole douce. France. PLACE NE.

Instituto Geológico y Cartográfico de Cataluña. www.icgc.cat

Koolhaas, Rem.; (2006). Junkspace. Quodlibet. ISBN: 978-8874621125

Lynch, Kevin (1960). The Image of the City. U.S.A. MIT Press.

Pérez, Y. (25 de agosto de 2017). Fer Feina a L'Ortoll. Diari de Vilanova.

Redacción. (07 de diciembre de 2017). De la montaña a la playa: el particular viaje de un grupo de caballos en trashumancia. Lavanguardia.com.

Tschumi, Bernardt. (1987). Parc de la Villette. 